\title{
Isolation and Functional Analysis of the Regulation of Branching by Isopentenyl Transferase Gene CmIPT1 in Chrysanthemum morifolium cv. 'Jinba'
}

\author{
Abdurazak Ishak1, Lili Dong², Huang Rong², Shuiming Zhang², Liangjun Zhao1* \\ ${ }^{1}$ Department of Ornamental Horticulture and Landscape Architecture, China Agricultural University, Beijing, China \\ ${ }^{2}$ Department of Ornamental Horticulture, Anhui Agricultural University, Hefei, China \\ Email: *471544710@qq.com
}

How to cite this paper: Ishak, A., Dong, L., Rong, H., Zhang, S. and Zhao, L. (2018) Isolation and Functional Analysis of the Regulation of Branching by Isopentenyl Transferase Gene CmIPT1 in Chrysanthemum morifolium cv. 'Jinba'. American Journal of Molecular Biology, 8, 92-101. https://doi.org/10.4236/ajmb.2018.82008

Received: December 19, 2017

Accepted: April 10, 2018

Published: April 13, 2018

Copyright $\odot 2018$ by authors and Scientific Research Publishing Inc. This work is licensed under the Creative Commons Attribution International License (CC BY 4.0).

http://creativecommons.org/licenses/by/4.0/

CC) (i) Open Access

\begin{abstract}
Today's chrysanthemums are highly evolved flowering plants and they are considered as one of the most important ornamental cut flowers. In this research an isopentenyl transferase gene named CmIPT1 was isolated from Chrysanthemum morifolium cV. 'Jinba' using RACE and RT-PCR methods. The full cDNA sequence of CmIPT1 was $873 \mathrm{bp}$ which encoded a deduced protein of 290 amino acids. It contained GxxGxGKS which is a conserved sequence of the typical domain of IPT family. The phylogenetic tree analysis of CmIPT1 in Chrysanthemum morifolium cv. 'Jinba' shows that it has the closest relationship with CcIPT1 in Cynara cardunculus var. scolymus. Expression of $C m I P T 1$ was higher in stems and apex, whereas it was lower in leaves and roots. And the overexpression of $C m I P T 1$ obviously increased the number of rosette branches in Arabidopsis. Here, in our study, we showed that CmIPT1 is a positive regulator of branch development in Chrysanthemum and may play a key role in regulating lateral branch formation of Chrysanthemum plants.
\end{abstract}

\section{Keywords}

Chrysanthemum, Cytokinin, CmIPT1, Shoot Branching

\section{Introduction}

The development of branching in plants involves the formation and subsequent growth of axillary buds [1]. Plant hormones, location on the plant, gene expression and environment all influence the growth of axillary buds. Hormones are 
one of the primary factors regulating branching. At present, it has been considered that three types of hormones participate in regulating the growth of axillary buds. Cytokinins accelerate the growth of axillary buds [2], while auxin and strigolactones inhibit the growth of axillary buds [3] [4] [5] [6]. Cytokinin is considered to be the second messenger [7], and influences the growth of axillary buds under mutual antagonization with strigolactones [8]. Isopentenyl transferase (IPT) is the first reaction in the biosynthesis of cytokinin, and is also a limiting enzyme in cytokinin biosynthesis. There are two types of Isopentenyltransferase (IPT), namely tRNA-IPT which forms the cis-form zeatin, and adenylate isopentenyltransferase (A-IPT) which forms isopentenyladenine as well as anti-form zeatin [9]. The IPT family of genes has been identified in Arabidopsis [10], rice [11], pea [12] and corn [13]. However, $I P T 1$ is rarely studied. Research on Physcomitrella patens showed that IPT1 was the main enzyme which catalysed the prenylation of tRNA, and mutation of IPT1 increased the number of lateral branches [14]. Research showed that the level of expression of PSIPTI and PSIPT2 in peas increased significantly and axillary bud started germinating after removing the shoot top by pruning. This illustrated that cytokinin was synthesized promptly to accelerate the growth of axillary buds after removing the shoot top by pruning [15]. According to the research results, IPT1 plays an important role in regulating lateral branch formation and the development of plants.

Chrysanthemum is native to China, with a long history of cultivation and abundant cultural connotations, it is considered as one of the most important ornamental cut flowers. Plant architecture is one of the important factors which needs to be regulated when cultivating Chrysanthemum. In this study, we cloned an IPT1 homologous gene called CmIPT1 from C. morifolium cv. 'Jinba', and studied its expression pattern using fluorescent quantitative PCR. We also studied the role of the CmIPT1 gene in regulating branching by using allogenic transformation of Arabidopsis with the aim of breeding new varieties of cut-flower Chrysanthemum having less lateral branches or without any lateral branches by means of molecular biology techniques.

\section{Materials and Methods}

\subsection{Materials}

In this study, we used Chrysanthemum morifolium cv. 'Jinba' from the Germplasm Nursery of Anhui Agricultural University. Chrysanthemum cuttings and Arabidopsis wild-type (col-0) as well as transgenic Arabidopsis were all grown in a culture room at a temperature of $(20 \pm 2)^{\circ} \mathrm{C}$, photoperiod of $16 \mathrm{~h} / 8 \mathrm{~h}$ (light/dark), illumination intensity of $100-120 \mu \mathrm{mol} \cdot \mathrm{m}^{-2} \cdot \mathrm{s}^{-1}$.

\subsection{Method}

\subsubsection{Cloning of CmIPT1}

Total RNA of Chrysanthemum was extracted from stems using Trizol reagent. Residual DNA was digested using RNase-free DNase. cDNA anti sense was syn- 
thesized by using M-MLV reverse transcriptase. Degenerate primers were designed according to the conservative nucleotide sequence of IPT1 in Cynara cardunculus var. scolymus, Medicago truncatula, Lotus japonicus and Arabidopsis thaliana. An intermediate fragment was obtained by amplification with degenerate primers. Specific primers for 5' and 3' were designed according to the sequence of the intermediate fragment. The 5' and 3' terminal sequences of CMIPT1 were obtained using a SMART ${ }^{\mathrm{er}} \mathrm{TM}$ RACE cDNA Amplification Kit. DNAMAN 6.0 splices joints tripartite sequences, and finally the full length sequence of the CmIPT1 gene was obtained. CmIPT1 was amplified using TransStart Fast Pfu polymerase and was connected with the pEASY-Blunt Simple vector. DNA sequencing was conducted by Sangon Biotech (Shanghai). PCR amplification reaction procedure and conditions were: $95^{\circ} \mathrm{C}$ initial denaturation 5 min; $95^{\circ} \mathrm{C}$ denaturation $30 \mathrm{~s}, 55^{\circ} \mathrm{C}$ annealing $30 \mathrm{~s}, 72^{\circ} \mathrm{C}$ extension $2 \mathrm{~min}$, overall 34 cycles; finally $72^{\circ} \mathrm{C}$ extension $10 \mathrm{~min}, 12^{\circ} \mathrm{C}$ preserved (Table 1 ).

Table 1. Primers used in this study.

\begin{tabular}{ccc}
\hline Primers & Sequence 5'-3' & Usage \\
\hline IPT1-PF & CTCCGACAAAATGCAAGTCTAC & Amplification of CmIPT1 middle fragment \\
IPT1-PR & TTCACAATCT TTACGCTTGG & Amplification of CmIPT1 middle fragment \\
IPT1-5'-R1 & CGACCCGAGACTCCGAAAATCCGAG & Amplification of CmIPT1 5' end \\
IPT1-5'-R2 & GGGTCGGGTCAATGGCTCCGAGAAG & Amplification of CmIPT1 5' end \\
IPT1-3'-F1 & CACGTGTCAGTTGGCGAAAAGACAAATAGG & Amplification of CmIPT1 3' end \\
IPT1-3'-F2 & GAGTGGATGCCACCGAGGCGTTTAA & Amplification of CmIPT1 3' end \\
IPT1-RTPF & CGCCAAGTTCTTTCCCGAGTATCC & qRT-PCR for CmIPT1 \\
IPT1-RTPR & ATGCCAAGGTCTCTGAGCTTCTGC & qRT-PCR for CmIPT1 \\
18S-F & CTCATGGGATGTGGCTTCTT & Reference gene in Chrysanthemum \\
18S-R & GCGTTCAAAAACTCGATGGT & Reference gene in Chrysanthemum \\
Tubulin-F & GAGCCTTACAACGCTACTCTGTCTGTC & Reference gene in Arabidopsis \\
Tubulin-R & ACACCAGACATAGTAGCAGAAATCAAG & Reference gene in Arabidopsis \\
\hline
\end{tabular}

2.2.2. CmIPT1 Sequence Analysis and Construction of a Polygenetic Tree Multiple comparison analyses were conducted between the protein sequences of gene code and homologous sequences of other species in the NCBI data base, using clustalX 1.8 and MEGA 5.05 software to establish maximum likelihood phylogenetic tree, adopting the neighbor-joining method [16], and by setting up bootstrap analysis repeated 1000 times.

\subsubsection{Expression Analysis of CmIPT1 from Different Tissues of Chrysanthemum and after Removing the Shoot Top by Pruning}

RNA was extracted from root, stem, leaf and apex of the plant respectively, using $2 \mu \mathrm{g}$ of each, and after DNase digestion, cDNA template was obtained by conducting reverse transcription using M-MLV reverse transcriptase. Real-time fluorescent quantitative PCR (qRT-PCR) was used, adopting the ABI 7500 Fast 
real-time PCR system and SYBR Premix Ex Taq ${ }^{\text {Tw }}$ II fluorochrome. The response procedure as follows: $95^{\circ} \mathrm{C}$ denaturation $90 \mathrm{~s}, 95^{\circ} \mathrm{C} 15 \mathrm{~s}, 60^{\circ} \mathrm{C} 30 \mathrm{~s}, 40$ cycles. Data analysis was conducted using 7500 software (version 2.0.4). Tubulin was used as the reference gene, and the $2^{-\Delta \Delta C T}$ method was used to compute relative expression [17].

\subsubsection{Construction of a CmIPT1 Overexpression Vector}

Sal I and HindW cutting sites were respectively added in the upstream and downstream primer, CmIPT1. The full length sequence was amplified using M-MLV and ligated into the PJL-Blue vector. Sequencing was conducted by Sangon Biotech (Shanghai). The correct plasmid from sequencing was ligated into the PFK205 expression vector through LR reaction.

\subsubsection{Gene Transfer and Phenotypic Analysis}

The vector plasmid was transformed into (Agrobacterium tumefaciens) ASE, and was then transformed into Arabidopsis thaliana wild type col-0 using the floral dip method [18]. Transformants were selected using 1/2 MS which contained $50 \mathrm{mg} \cdot \mathrm{L}^{-1}$ kanamycin, and selected $\mathrm{T} 3$ generation transgenic plants were transplanted into pots. RNA was extracted from rosette leaves of the control and T3 generation transgenic plants respectively. cDNA was synthesized using reverse transcription and the level of expression of $C m I P T 1$ was determined. The number of lateral branches and plant height were recorded after culturing the plant for 40 days in growth chamber.

\section{Results and Analysis}

\subsection{Cloning Full Length cDNA of the CmIPT1 Gene from Chrysanthemum}

Based on the available IPT1 sequences from plant species such as Brassica campestris, Arabidopsis thaliana, Raphanus sativus and Solanum tuberosum, a 781 bp length DNA fragment was obtained from Chrysanthemum morifolium using PT1-PF/IPT1-PR. Amplification of the 5' end of the CmIPT1 gene was conducted using the gene-specific primer IPT1-5'F1/IPT1-5'R1, and a fragment with the length of $477 \mathrm{bp}$ was obtained (Figure 1(a)). Amplifications of the 3' end sequence of the CmIPT1 gene were conducted using 3' Gene Racer outer and inner primers respectively with IPT1-3'F1 and IPT1-3'F2, and finally a $588 \mathrm{bp}$ fragment was obtained with a poly A tail (Figure 1(b)). The 1615 bp CmIPT1 gene sequence was obtained by joining the intermediate fragment, the 3 ' end sequence and the 5 ' end sequences.

\subsection{Sequence Analysis}

The CmIPT1 gene with an overall length of 1155 bp was extracted from Chrysanthemum using the RACE method, and this gene encoded a protein with 384 amino acids. The predicted molecular weight was $43.41 \mathrm{kD}$ and the theoretical isoelectric point was 8.68, using ExPaSy ProtParam online tool. The amino acid 


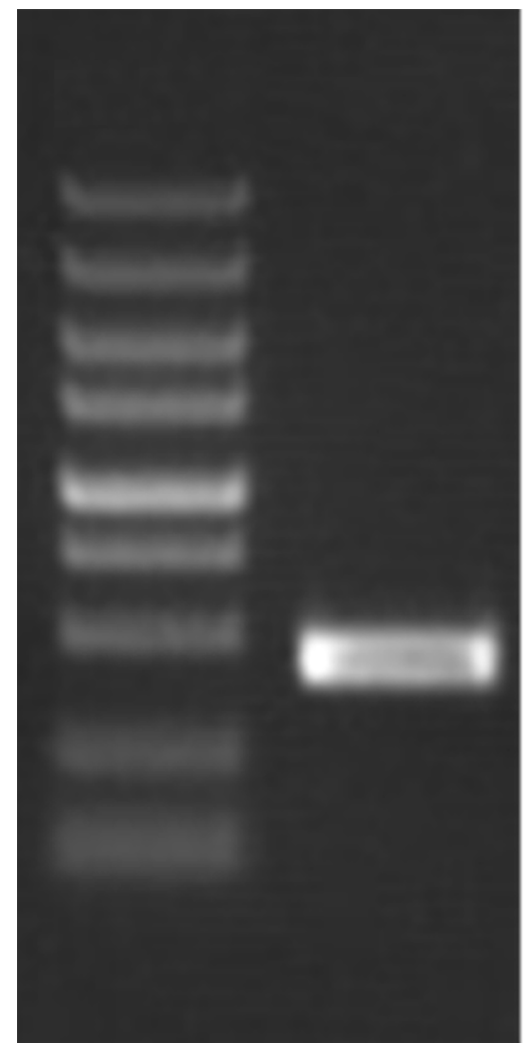

(a)



(b)

Figure 1. The amplification of CmIPT1. (a) Amplification results of 5'RACE; (b) Amplification results of 3'RACE.

sequence coded by the CmIPT1 gene (Figure 2) was compared with amino acid sequences of other plant species, and the result showed that CmIPT1 had the binding site GxxGxGKS sequence motif of ATP/ADP/AMP and DMAPP, which is the conserved domain of all IPT genes [10].

\subsection{Phylogenetic Analyses of the IPT1 Homologous Protein}

To confirm the genetic relationship between CmIPT1 and IPT1 from other plant species, multiple sequence alignment and phylogenetic analyses were conducted between CIIPT1 and IPT1 which had already been cloned from eight plant species such as Cynara cardunculus var. scolymus, Lotus japonicus, Arabidopsis thaliana, Morus notabilis, Malus domestica, Medicago truncatula, Brassica napus and Solanum tuberosum using clustal $\times 1.8$ and MEGA 5.05 software (Figure 3). The results showed that the origin of $C m I P T 1$ and $I P T 1$ from other plant species was the same, and was separated together with the other plant species during periods of evolution. CmIPT1 was most similar to CCIPT1 of Cynara cardunculus, and was most distant from StIPT1 of potato.

\subsection{Gene Expression Analysis of CmIPT1 in Different Tissues of Chrysanthemum}

To reveal the expression features of CmIPT1 gene in Chrysanthemum, the 


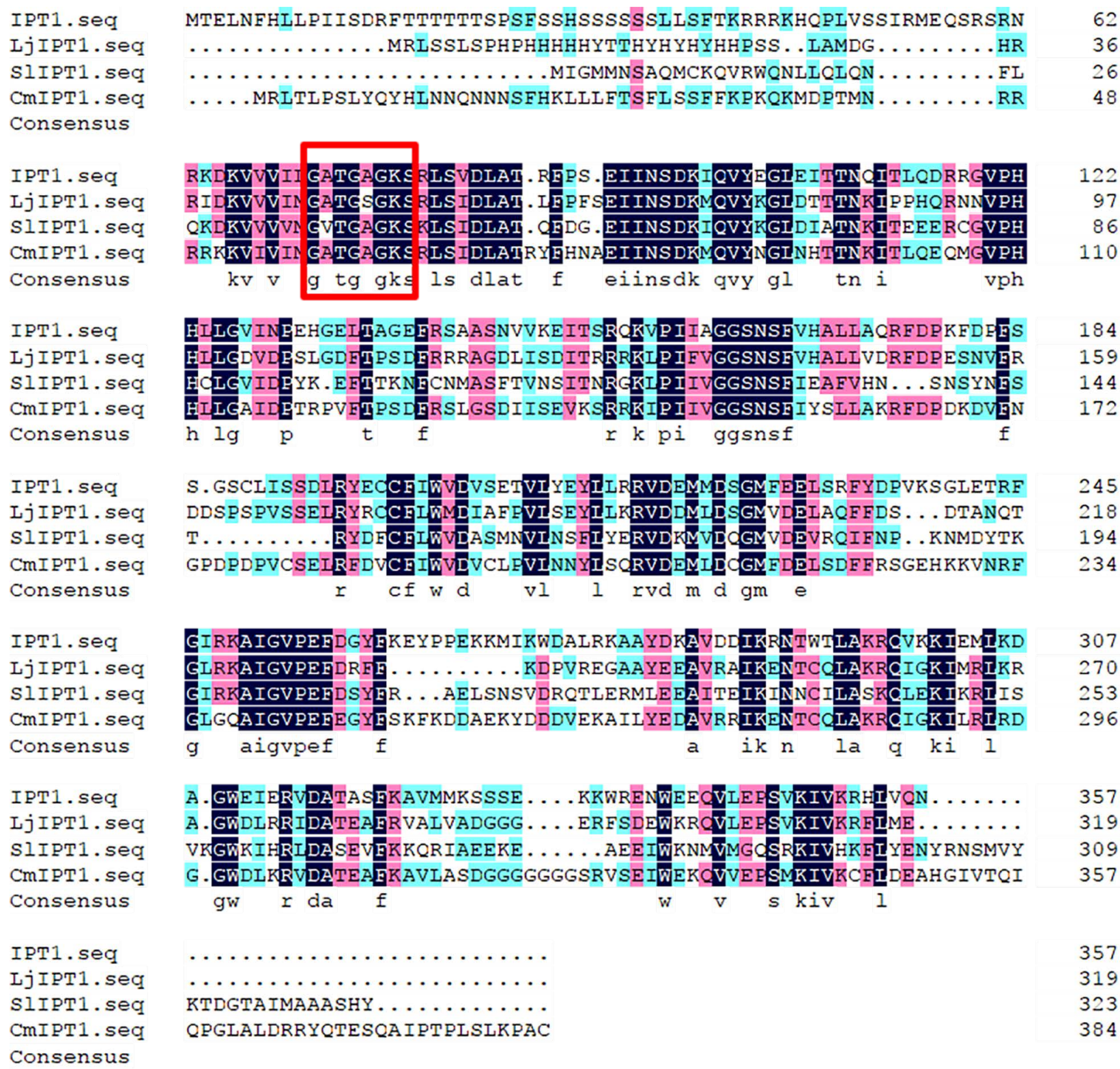

Figure 2. Alignment of the predicted amino acid sequences of Chrysanthemum CmIPT1 compared with Arabidopsis thaliana (AtIPT1), Lotus japonicus (LjIPT1) and Solanum lycopersicum (SIIPT1). Note: Black, red, and blue stands for completely conserved, partially conserved, and similar amino acids respectively. The conserved sequence is highlighted.

expression of CmIPT1 in different tissues of C. morifolium cv. 'Jinba' was detected. The results indicated that $C m I P T 1$ gene was expressed in roots, stems, leaves and the apex of the plant. Expression of CmIPT1 in stem and apex was relatively high, while the expression in root and leaf tissues was relatively low (Figure 4).

\subsection{Phenotypic Analysis of CmIPT1 Over-Expressed Arabidopsis thaliana}

To construct a plant expression vector based on $C m I P T 1$, the gene was transformed into wild type Arabidopsis, and six transgenic plants were obtained. 


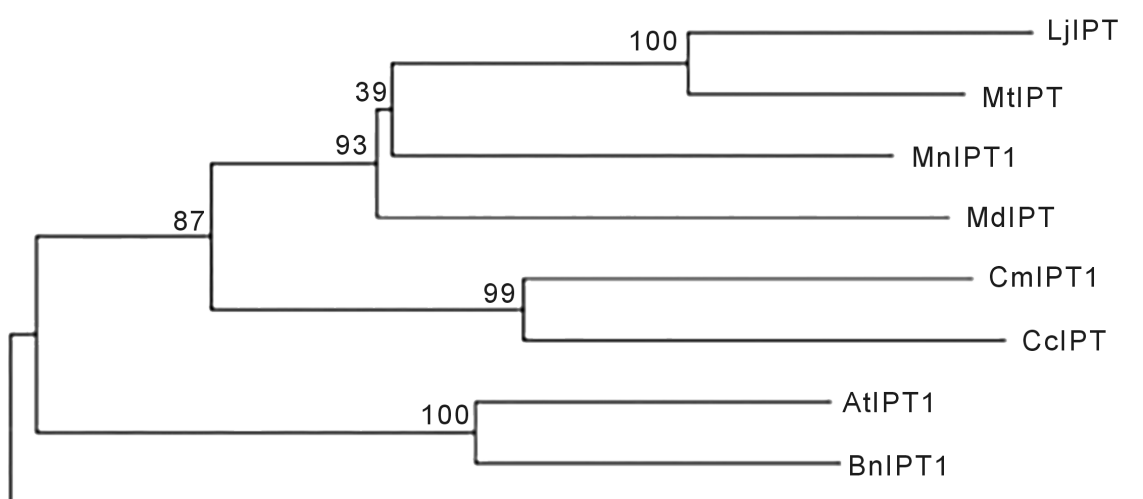

StIPT1

$$
0.05
$$

Figure 3. Phylogenetic analysis of IPT from different plant species. The sequence accession numbers of IPT from the different plant species are as follows: Lotus japonicus LjIPT (ABD93932), Medicago truncatula MtIPT (XP_00359265 3), Morus notabilis MnIPT1 (XP_010099238), Malus domestica MdIPT (ADY8 0561), Cynara cardunculus var. scolymus CcIPT1 (KVH96285), Arabidopsis thaliana AtIPT1 (B AB59040), Brassica napus BnIPT1 (XP_013691077), Solanum tuberosum StIPT1 (XP_006360459).

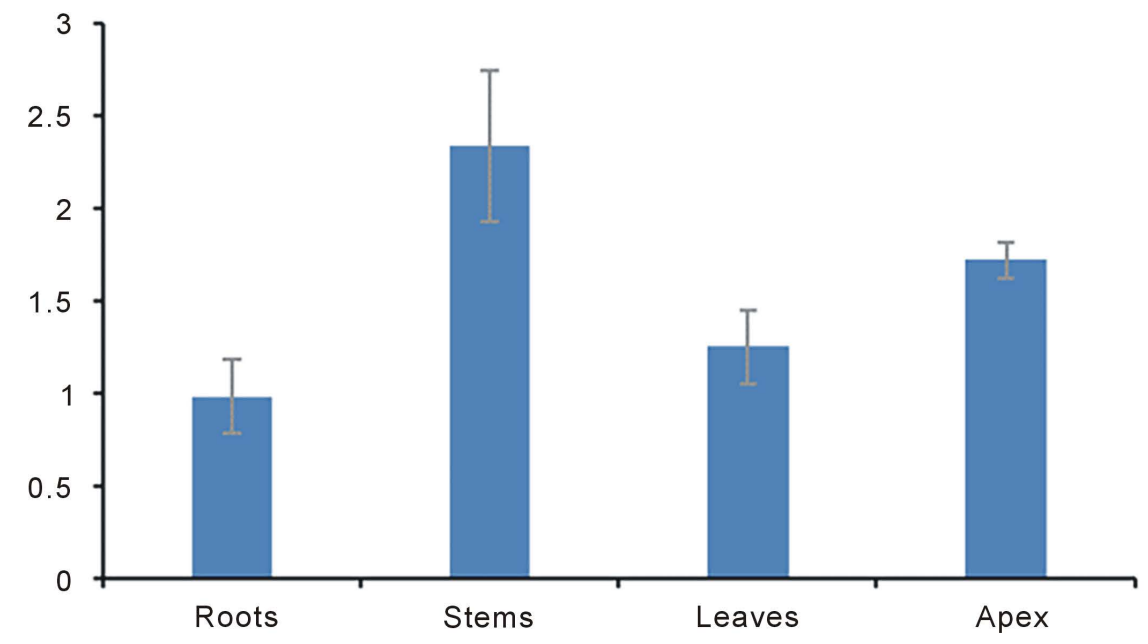

Figure 4. The relative expression of $C m I P T 1$ in different tissues of $C$. morifolium cv. 'Jinba'.

CMIPT1 gene expression was detected in randomly selected transgenic plants. After 40 days growth, the number of lateral branches in wild type and transgenic plants was 3.5 and 6.3 respectively, with the number of lateral branches in transgenic plants being significantly greater. The mean heights of wild type and transgenic plants were 25.2 and $17.0 \mathrm{~cm}$, respectively, with the transgenic plants being significantly shorter (Figure 5 ).

\section{Discussion}

Chrysanthemum is one of world's four major cut flowers. However, cut flower Chrysanthemums generally have many lateral branches. Controlling lateral 
(a)

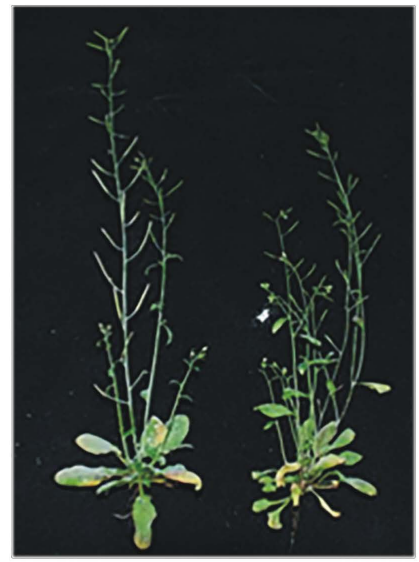

WT

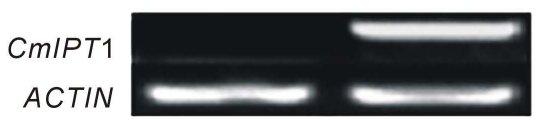

(b)

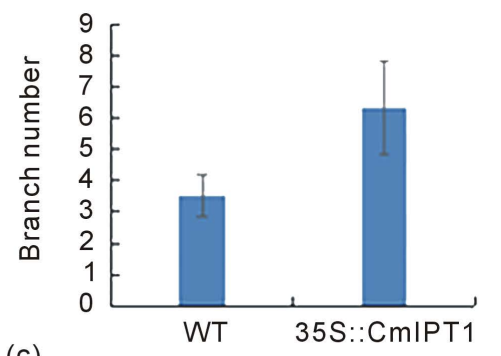

(c)

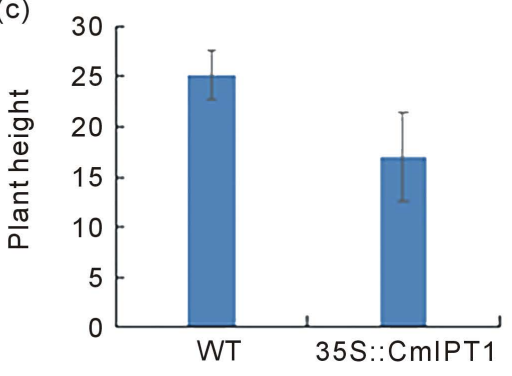

Figure 5. Comparison of number of branches and plant height of wild type Arabidopsis and transformants carrying 35S: CmIPT1. (a) Phenotype of wild type Arabidopsis and transformants carrying 35S::CmIPT1; (b) Branch number per plant of wild type Arabidopsis and transformants with $35 \mathrm{~S}: \mathrm{CmIPT1}$; (c) Plant height of wild type Arabidopsis and transformants with 35S::CmIPT1.

branching using hormones has been very difficult, and doing it manually is a common practice, but is very time-consuming. For C. morifolium cv. 'Jinba', for instance, the cost of manual bud removal is $1 / 3$ of the overall production cost. So, producing new varieties of Chrysanthemum with no lateral branches or with fewer lateral branches is the key to promoting the efficient production of this flower. In our study, a homologous gene of IPT1 from C. morifolium cv. 'Jinba' was cloned. The predicted amino acid sequence of the gene contains the conserved structural domain of IPT family genes, GATGAGKS, hence it was named $C m I P T 1$. Phylogenetic tree analysis indicated that the origin of $C m I P T 1$ and IPT1s from other plants was similar. The Chrysanthemum IPT1 gene CMIPT1 was most closely related to that of Cynara cardunculus, and was most distant from those of Solanum tuberosum and Lotus japonicus. Asteraceae family contains both Chrysanthemum and Cynara. So, some degree of closer relatedness is to be expected. After testing gene expression levels in root, stem, leaf, and apex of the Chrysanthemum plant, it was found that the CmIPT1 gene was expressed in all 4 different tissues, and the expression in stem and apex of the plant was relatively high while the expression was relatively low in root and leaf of the plant.

Direct application of cytokinin could accelerate the growth of axillary buds, and synthesis of cytokinin is controlled by auxin, so cytokinin is considered to be the second messenger of auxin [7]. The CmIPT1 gene was transformed into wild type Arabidopsis, and the number of lateral branches in transgenic plants was increased significantly, showing that that $C m I P T 1$ could be the key gene 
that controls lateral branch development in Chrysanthemum and to produce single-stemmed plants a knockout mutant of CmIPT1 can be developed and tested.

\section{Conclusion}

In summary, after analyzing the expression features and functional analysis of CmIPT1 gene, it was found that there is a correlation between the expression of $C m I P T 1$ and the number of lateral branches, and it therefore appears to be an important regulator of lateral branch development in Chrysanthemum. The CIIPT1 gene is worthy of further study, in order to provide insight into the possibility of breeding new varieties of cut-flower Chrysanthemum using molecular biology.

\section{References}

[1] McSteen, P. and Leyser, O. (2005) Shoot Branching. Annual Review of Plant Biolo$g y$, 56, 353-374. https://doi.org/10.1146/annurev.arplant.56.032604.144122

[2] Cline, M.G. (1991) Apical Dominance. The Botanical Review, 57, 318-358. https://doi.org/10.1007/BF02858771

[3] Thimann, K.V. and Skoog, F. (1933) Studies on the Growth Hormone of Plants. The Inhibiting Action of the Growth Substance on Bud Development. Proceedings of the National Academy of Sciences of the United States of America, 19, 714-716. https://doi.org/10.1073/pnas.19.7.714

[4] Thimann, K.V. and Skoog, F. (1934) On the Inhibition of Bud Development and Other Functions of Growth Substance in Vicia faba. Proceedings of the National Academy of Sciences of the United States of America, 114, 317-339. https://doi.org/10.1098/rspb.1934.0010

[5] Gomez-Roldan, V., Fermas, S., Brewer, P.B., et al. (2008) Strigolactone Inhibition of Shoot Branching. Nature, 455, 189-194. https://doi.org/10.1038/nature07271

[6] Umehara, M., Hanada, A., Yoshida, S., et al. (2008) Inhibition of Shoot Branching by New Terpenoid Plant Hormones. Nature, 455, 195-200. https://doi.org/10.1038/nature07272

[7] Brewer, P.B., Dun, E.A., Ferguson, B.J., et al. (2009) Strigolactone Acts Downstream of Auxin to Regulate Bud Outgrowth in Pea and Arabidopsis. Plant Physiology, 150, 482-493. https://doi.org/10.1104/pp.108.134783

[8] Dun, E.A., Germain, A.D.S., Rameau, C. and Beveridge, C.A. (2012) Antagonistic Action of Strigolactone and Cytokinin in Bud Outgrowth Control. Plant Physiology, 158, 487-498. https://doi.org/10.1104/pp.111.186783

[9] Kaori, M., Petr, T., Miho, M.K., Tomohiko, K., Shusei, S., Danuse, T., Satoshi T., Goran, S. and Tatsuo, K. (2006) Roles of Arabidopsis ATP/ADP Isopentenyltransferases and tRNA Isopentenyltransferases in Cytokinin Biosynthesis. Proceedings of the National Academy of Sciences of the United States of America, 103, 16598-16603. https://doi.org/10.1073/pnas.0603522103

[10] Takei, K., Sakakibara, H. and Sugiyama, T. (2001) Identification of Genes Encoding Adenylate Isopentenyltransferase, a Cytokinin Biosynthesis Enzyme, in Arabidopsis thaliana. Journal of Biological Chemistry, 276, 26405-26410. https://doi.org/10.1074/jbc.M102130200

[11] Hirose, N., Takei, K., Kuroha, T., Kamada-Nobusada, T., Hayashi, H. and Sakaki- 
bara, H. (2008) Regulation of Cytokinin Biosynthesis, Compartmentalization and Translocation. Journal of experimental Botany, 59, 75-83. https://doi.org/10.1093/jxb/erm157

[12] Ye, C.J., Wu, S.W., Kong, F.N., Zhou, C.J., Yang, Q.K, Sun, Y. and Wang, B. (2006) Identification and Characterization of an Isopentenyltransferase (IPT) Gene in Soybean (Glycine max L.). Plant Science, 170, 542-550. https://doi.org/10.1016/j.plantsci.2005.10.008

[13] Brugière, N., Humbert, S., Rizzo, N., Bohn, J. and Habben, J.E. (2008) A Member of the Maize Isopentenyl Transferase Gene Family, Zea mays Isopentenyl Transferase 2 ( $Z m I P T 2)$, Encodes a Cytokinin Biosynthetic Enzyme Expressed during Kernel Development. Plant Molecular Biology, 67, 215-229.

https://doi.org/10.1007/s11103-008-9312-x

[14] Lindner, C.A., et al. (2014) Isopentenyltransferase-1 (IPT1) Knockout in Physcomitrella Together with Phylogenetic Analyses of IPTs Provide Insights into Evolution of Plant Cytokinin Biosynthesis. Journal of Experimental Botany, 65, 2533-2543. https://doi.org/10.1093/jxb/eru142

[15] Tanaka, M., Takei, K., Kojima, M., Sakakibara, H. and Mori, H. (2006) Auxin Controls Local Cytokinin Biosynthesis in the Nodal Stem in Apical Dominance. The Plant Journal, 45, 1028-1036. https://doi.org/10.1111/j.1365-313X.2006.02656.x

[16] Saitou, N. and Nei, M. (1987) The Neighbor-Joining Method: A New Method for Reconstructing Phylogenetic Trees. Molecular Biology and Evolution, 4, 406-425.

[17] Livak, K.J. and Schmittgen, T.D. (2001) Analysis of Relative Gene Expression Data Using Real Time Quantitative PCR and the 2-CT Method. Methods, 25, 402-408. https://doi.org/10.1006/meth.2001.1262

[18] Clough, S.J. and Bent, A.F. (1998) Floral Dip: A Simplified Method for Agrobacterium-Mediated Transformation of Arabidopsis thaliana. The Plant Journal, 16, 735-743. https://doi.org/10.1046/j.1365-313x.1998.00343.x 